\title{
Scanning Gel Electrochemical Microscopy (SGECM): Lateral physical resolution by current and shear force feedback
}

\author{
Ning Dang, Mathieu Etienne, Alain Walcarius, Liang Liu* \\ Laboratoire de Chimie Physique et Microbiologie pour les Matériaux et l'Environnement \\ (LCPME), UMR 7564, CNRS-Université de Lorraine, Villers-lès-Nancy 54600, France
}

\begin{abstract}
Scanning gel electrochemical microscopy (SGECM) is a novel technique measuring local electrochemistry based on a gel probe. The gel probe, which is fabricated by electrodeposition of hydrogel on a microdisk electrode, immobilizes the electrolyte and constitutes a two-electrode system upon contact with the sample. The contact area determines the lateral physical resolution of the measurement, and considering the soft nature of the gel it is essential to be well analyzed. In this work, the lateral physical resolution of SGECM is quantitatively studied from two aspects: (1) marking single sampling points by locally oxidizing $\mathrm{Ag}$ to $\mathrm{AgCl}$ and measuring their size; (2) line scan over reference samples with periodic topography and composition. The gel probe is approached to the sample by either current or shear force feedback, and the physical resolution of them is compared. For the optimal gel probe based on $25 \mu \mathrm{m}$ diameter Pt disk electrode of $\mathrm{R}_{\mathrm{g}} \approx 2$, the lateral physical resolution of SGECM at contact position is $c a .50 \mu \mathrm{m}$ for current feedback and $c a .67 \mu \mathrm{m}$ for shear force feedback. More importantly, the lateral physical resolution of SGECM can be flexibly tuned in the range of 14 to $78 \mu \mathrm{m}$ by pulling or pressing the gel probe after touching the sample. In general, current feedback is more sensitive to gel-sample contact than shear force feedback. But the latter is more versatile, which is also applicable to non-conductive samples.
\end{abstract}

Key words: SGECM, physical resolution, patterning, current feedback, shear force feedback

Scanning electrochemical probe techniques are powerful tools for analysing local electrochemical behaviour of interfaces between electrolyte and various materials, such as metals ${ }^{1,2}$, metal oxides ${ }^{3-7}$, electrocatalysts ${ }^{8-10}$, biological species ${ }^{11-14}$ as well as minerals ${ }^{15,16}$. Among these techniques, scanning electrochemical microscopy (SECM) and scanning ion conductance microscopy (SICM) are the most developed, with the concept of using a micro-/nano- electrode or capillary scanning over a sample immersed in an electrolyte. Recently, scanning droplet cell techniques (SDC), such as scanning electrochemical cell microscopy (SECCM), are also becoming popular. The concept is to measure local electrochemistry with a localized electrolyte, which is a droplet coming out from a pulled capillary that is in local contact with the sample surface.

Different from the techniques above, Scanning Gel Electrochemical Microscopy (SGECM) is a novel approach based on a gel probe. It uses the microelectrode as in SECM, but instead of 
scanning the microelectrode in electrolyte, the electrolyte is localized in a gel that is deposited on the microelectrode. During the measurement, the gel probe is approached to be in contact with the sample, allowing local electrochemistry to be measured. The electrolyte is immobilized in the gel probe, offering the following advantages: (1) The sample can be exposed in air; (2) The uncontrollable spreading of electrolyte over the sample surface can be inhibited ${ }^{17}$; (3) The soft contact between the gel and the sample may protect the sample from accidental scratching. In our previous studies, hemispherical gel probes were fabricated by electrochemical polymerization of chitosan on Pt or Ag micro-disk electrodes ${ }^{18,19}$. The probes were approached to the samples by shear force feedback, and the lateral scan was achieved in tapping mode. The topography of samples was mapped from the touching position. At the same time, amperometric or potentiometric analysis were carried out for mapping the current (under fixed or scanning potential) or open circuit potential, respectively.

Like other scanning probe microscopies, SGECM also has a key issue of resolution, especially lateral resolution, as it determines the quantification and applications. The lateral resolution has two aspects, scan resolution and physical resolution. The former is determined by the movement of control units (motor or piezo), while the latter refers to the sampling area of a single pixel measurement. With modern instrumentation, the scan resolution can easily reach $0.1 \mathrm{~nm}$, but the physical resolution is usually much lower. Thus, the lateral resolution of SGECM is limited by the physical resolution. Since SGECM is a contact method, the physical resolution is essentially determined by the contact area. Moreover, due to the soft nature of the gel, one may expect that the contact area depends on the force of contact. That is to say, the lateral resolution may be tuned by squeezing or stretching the gel after touching the sample. Therefore, it is highly desired to quantitatively determine the lateral physical resolution of SGECM.

In this work, two methods are presented to quantitatively study the lateral physical resolution of SGECM. One method is based on marking single sampling points and measuring their size. This is achieved by locally oxidizing $\mathrm{Ag}$ to $\mathrm{AgCl}$ with the gel probe. The color change leaves a permanent mark for the contact area, which indicates the lateral physical resolution. Another method is the line scan over a sample with periodic topography and composition. The profile of the sample is measured by the gel probe and a commercial profilometer (as reference). By comparing the results, the lateral physical resolution is analyzed. Apart from the shear force feedback used in previous works, current feedback is also used for approaching the gel probe to conductive samples. The lateral resolution of the two feedback protocols is compared. It is seen that current feedback is more sensitive to gel-sample contact than shear force feedback, but the latter is more versatile regardless of the sample conductivity.

\section{Experimental Section}

SGECM Setup: SGECM measurements were carried out on a home-made setup. Three-dimensional positioning of the gel probe was controlled by a step motor system (Owis, 
Staufen, Germany). In addition, vertical positioning in $z$ axis is more accurately controlled by a piezo in closed-loop mode with position encoder (Physik Instrumente, Karlsruhe, Germany). Electrochemical measurements were carried out with a PalmSens ${ }^{3}$ potentiostat (Palm Instruments BV, Houten, The Netherlands). The current was recorded with a 16-bit data acquisition card (Measurement Computing, Norton, United States). Shear force detection was achieved with two piezoelectric plates mechanically attached to the glass shield of probe ${ }^{20,21}$. A Lock-in Amplifier (Model 7270 DSP, AMETEK, United States) was used to generate AC signals for actuating one piezoelectric plate and measure the response from another plate. All the instruments were controlled with an integrate SGECM program written by VB.net (can be shared upon request). An optical microscope was placed for visualizing the positioning and movement of the gel probe.

Gel probe preparation: The gel probes were fabricated by electrodepositing chitosan hydrogel on Pt micro-disk electrodes as reported in previous works ${ }^{18,19}$. Pt wires of $25 \mu \mathrm{m}$ diameter were sealed in sharpened capillaries by epoxy resin to prepare the micro-disk electrodes $\left(R_{g} \approx 2\right)$. These electrodes were used for electrodepositing chitosan in a three-electrode system, with a chlorinated Ag wire as quasi-reference electrode (QRE) and a Pt wire as counter electrode. The chitosan solution was prepared by dissolving $0.8 \mathrm{wt} \%$ chitosan (medium molecular weight, Aldrich) in 1:1 (vol. ratio) glycerol/deionized water containing $0.125 \mathrm{~mol} / \mathrm{L} \mathrm{NaCl}$. Desired volume of $1 \mathrm{~mol} / \mathrm{L} \mathrm{HCl}$ solution (VWR Chemicals) was added to make sure that chitosan fully dissolves, and then the $\mathrm{pH}$ of solution was adjusted to about 5.5 by $\mathrm{NaOH}$ solution. Typically, the electrodeposition was carried out by applying a constant potential of $-1.1 \mathrm{~V}$ ( $v s$. $\mathrm{Ag} / \mathrm{AgCl} \mathrm{QRE}$ ) for $420 \mathrm{~s}$ with the deposition current monitored. The gel probes prepared showed hemispherical shape (Figure S1).

Approaching and retracting the gel probes: The gel probes were initially positioned in air and not in contact with the sample, as verified by optical microscope. They were approached to the sample while measuring the current and/or shear force signals, and the contact was sensed by the current or shear force reaching a pre-set threshold. This constituted a closed-loop feedback. For current feedback, a voltage was applied on the gel probe versus the sample during approaching. The probe stopped when the current reached $\pm 0.1 \mathrm{nA}$. For shear force feedback, an optimized frequency was chosen for obtaining a stable signal, and the approaching stopped when the amplitude changed by $\pm 0.2 \%$. After the gel probe touched the sample, it was immediately triggered to retract away from the sample. The current and shear force signals were continuously recorded until the probe moved back to the initial position. In this work, the contact position (sensed by either current or shear force feedback) is defined as $z=0$. Positive $z$ represents further approaching the gel probe (squeezing the gel), and negative $z$ represents retracting the probe away from the sample (stretching the gel).

AgCl deposition on Ag substrate: Ag foil (99.9\%, ChemPUR, Karlsruhe, Germany) was cut to $10 \times 10 \times 0.125 \mathrm{~mm}^{3}$, polished with Alumina paste $(0.05 \mu \mathrm{m})$, cleaned with acetone by sonication and dried in air before being used as substrate for deposition. The gel probe was 
approached to be in contact with the Ag foil with current or shear force feedback, and then the probe was purposely further approached or retracted by a given distance. For deposition, a voltage of $1.2 \mathrm{~V}$ was applied on Ag foil versus the gel probe for $300 \mathrm{~s}$, allowing Ag to be chlorinated forming $\mathrm{AgCl}$. After deposition at one point, the probe was withdrawn rapidly (at maximum speed allowed by the piezo) to detach from the sample and then laterally moved by $150 \mu \mathrm{m}$ for the next point. The deposited $\mathrm{AgCl}$ spots were examined by optical microscope (Nikon, Japan) and also characterized by scanning electron microscopy (SEM) equipped with energy dispersive X-ray spectroscopy (EDS) (Hitachi FEG S4800).

\section{Line scan of periodically shaped samples:}

Two typical samples with periodic shape and composition were analyzed by SGECM with current and shear force feedback. Sample 1 was a TEM grid of $\mathrm{Cu}$ (50 mesh, bar width of $c a$. $80 \mu \mathrm{m}$, hole size of $c a .450 \mu \mathrm{m}$, Electron Microscopy China) stick on a flat Fe plate. It was conductive and served as a reference sample for the lateral resolution determination. Sample 2 was commercial Au interdigitated electrodes (DropSens, bands/gaps of $200 \mu \mathrm{m}$ ) with one electrode plated with $\mathrm{Cu}$. The electroplating was carried out at $-0.8 \mathrm{~mA}$ for $3600 \mathrm{~s}$ in a commercial solution from Aldrich. This yielded a sample with three different materials: $\mathrm{Cu}$ (height of $c a .10 \mu \mathrm{m}$, conductive), ceramic (flat, insulating) and $\mathrm{Au}$ (flat, conductive). It was used for demonstrating the versatility of shear force feedback SGECM. The profile of both samples was analyzed on a commercial profilometer (Brüker Dektak XT) by a cone-shape probe of $2 \mu \mathrm{m}$ radius at a scan resolution of $0.05 \mu \mathrm{m}$.

For SGECM line scan, the gel probe was approached to touch the sample by current or shear force feedback, then was positioned to the desired $z$ position and kept for $3 \mathrm{~s}$. This allowed a stable steady state current to be recorded. Afterwards, the probe was withdrawn to detach from the sample and then laterally moved for $2 \mu \mathrm{m}$ for the next measuring point. Sample 1 was measured at $0.5 \mathrm{~V}\left(E_{\text {sample }} v s . E_{\text {probe }}\right)$, and Sample 2 was measured at $1 \mathrm{~V}\left(E_{\text {sample }} v s . E_{\text {probe }}\right)$.

\section{Results and Discussion}

The first step of any scanning probe technique is approaching the probe close to the sample. In SGECM, the gel probe should be in contact with the sample surface for measuring local electrochemistry, and the contact position would reveal the topography of the sample. Considering its soft nature, the gel probe can be further pressed or stretched after touching the sample. This may affect the contact area thus influence the lateral physical resolution of the measurements. Therefore, it is essential to find sensitive detection of contact by studying the approaching/retracting behaviour of the gel probe.

\section{Approach and retract the gel probe}

Like in the previous works ${ }^{18,19}$, the gel probes were first approached to the sample with shear force feedback, as illustrated in Figure 1A. Figure 1B shows that the amplitude of signal is almost constant when the gel probe is far away from the sample, thus it is normalized to $100 \%$. The signal starts to change when the probe gets close or in contact with the sample. We 
arbitrarily selected $\pm 0.2 \%$ as the threshold for stopping the approaching. Afterwards, the gel probe is immediately retracted from the sample. It is seen that the signal further increases upon retraction, but it decreases after $c a$. $-5 \mu \mathrm{m}$. At $c a$. $-20 \mu \mathrm{m}$ the signal recovers almost to the initial value before touching (100\%). This phenomenon is qualitatively reproducible (Figure S2), perhaps due to the pressing and stretching of the gel during the approaching and retraction.

When approaching the gel probe to a conductive sample, current could also be monitored by applying a voltage between them. Figure $1 \mathrm{C}$ compares the variation of current (measured at $1.2 \mathrm{~V}$ ( $\left.E_{\text {sample }} v s . E_{\text {probe }}\right)$ ) and shear force signals when approaching the gel probe. It is clearly seen that the current sharply increases from 0 (with noise) to $c a .3 .3 \mathrm{nA}$ at one position, which indicates that the gel probe touches the sample. This position is normalized to $z=0$. At the same time, the shear force signal also starts to change, which can be explained by the contact. Nevertheless, the probe is further approached for $c a .8 \mu \mathrm{m}$ until the shear force amplitude reaches the threshold $( \pm 0.2 \%)$ for stopping the approaching. The results indicate that current feedback is much more sensitive to gel-sample contact than shear force feedback. It should be noted that the sensitivity of shear force feedback could be improved by reducing the threshold, but it would also increase the risk of stopping the approaching before touching ("fake contact") due to the noise and drift of the signal.
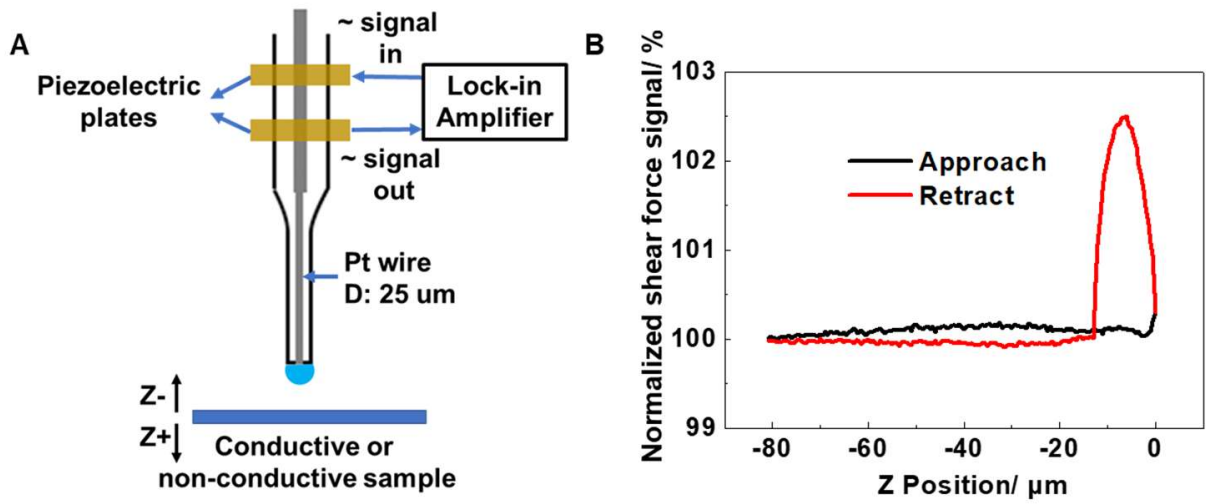

C

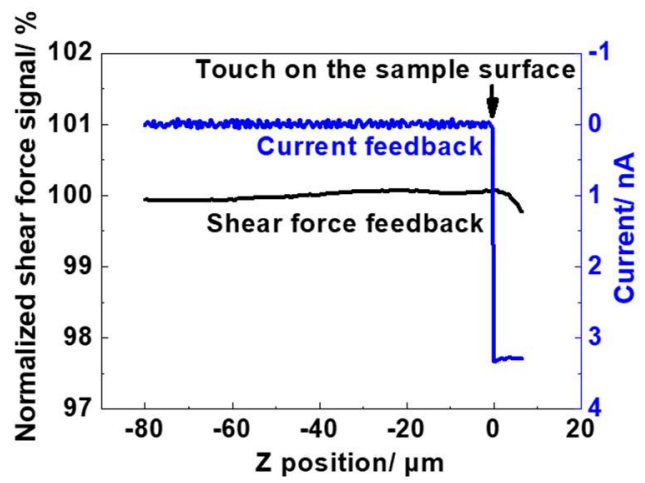

Figure 1 (A) Scheme of SGECM with shear force feedback; (B) Approach-retract curves of shear force feedback with the shear force threshold of $\pm 0.2 \%$; (C) Approach curves of shear force and current feedback. 
The sharp spike of current in Figure 1C is technically ideal for constructing a feedback loop. Therefore, we further studied the approaching and retracting behaviour of gel probes by current feedback under a constant applied voltage, as illustrated in Figure 2A. In principle, when the gel probe touches a conductive sample, any potential that is different from the open circuit potential (OCP) of the two-electrode system ( $\left.E_{\text {sample }} v s . E_{\text {probe }}\right)$ would give a current response. Therefore, even the OCP is unknown, the gel probe could still approach with an arbitrarily selected voltage. For example, current spikes are detected upon touching the gel probe with an Ag plate in a wide range of the applied voltage (Figure 2B). As the voltage increases, the current upon contact (the first point recorded with absolute value above a threshold that differentiates the noise) also increases, but even applying $\pm 1 \mathrm{mV}$ is sufficient for detecting the contact. Considering the time interval of data acquisition ( $c a .17 \mathrm{~ms})$, the current upon contact mainly reflects the non-Faradaic behaviour of the system. Moreover, the probes are immediately retracted from the sample after touching, and the current is monitored with the same voltage as approaching. Qualitatively, it is seen that the gel probe remains in contact with the sample after retracting for a certain distance. This can be explained by the stretching of the gel upon retraction that compensates its adhesion with the sample surface. Once the stretching force exceeds the adhesion, the gel detaches from the sample and the current response returns to zero (with noise). The detail current variation depends on the applied voltage. For example, at $\pm 1.2 \mathrm{~V}$ ( $E_{\text {sample }} v s$. $E_{\text {probe }}$ ) the current is almost unchanged up to $c a .-10 \mu \mathrm{m}$, and then decreases upon further retraction. At $c a .-16 \mu \mathrm{m}$, the current decreases to zero (with noise). The approach/retract behaviour is highly reproducible after a few approach-retract cycles (Figure S3), confirming that the gel probe undergoes elastic deformation and remains in contact with the sample by retraction up to $c a$. $-16 \mu \mathrm{m}$ (Figure 2C). It should be noted that the initial several approach-retract cycles may remove the loosely absorbed solvent in the gel. This step is practically important for quantitative measurements. At $\pm 0.5 \mathrm{~V}$, the current significantly decreases before reaching a plateau. Interestingly, after $c a$. $-11 \mu \mathrm{m}$ the current decreases again until returning zero at $c a$. $-16 \mu \mathrm{m}$. At $\pm 1 \mathrm{mV}$, the current gradually decreases until merging in noise at around $-10 \mu \mathrm{m}$. The difference in detaching position might indicate that the applied voltage affects the adhesion of the gel with the sample. Quantitative analysis of the retracting current is very complicated, because it is a two-electrode system affected by both non-Faradaic and Faradaic processes, as well as the change in the electrical resistance of the gel due to stretching. This will be further explored in future but it is beyond the focus of this work. 


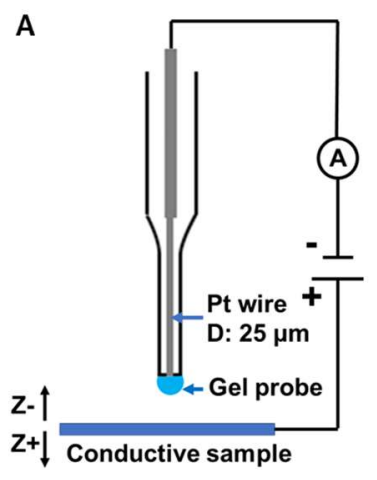

B
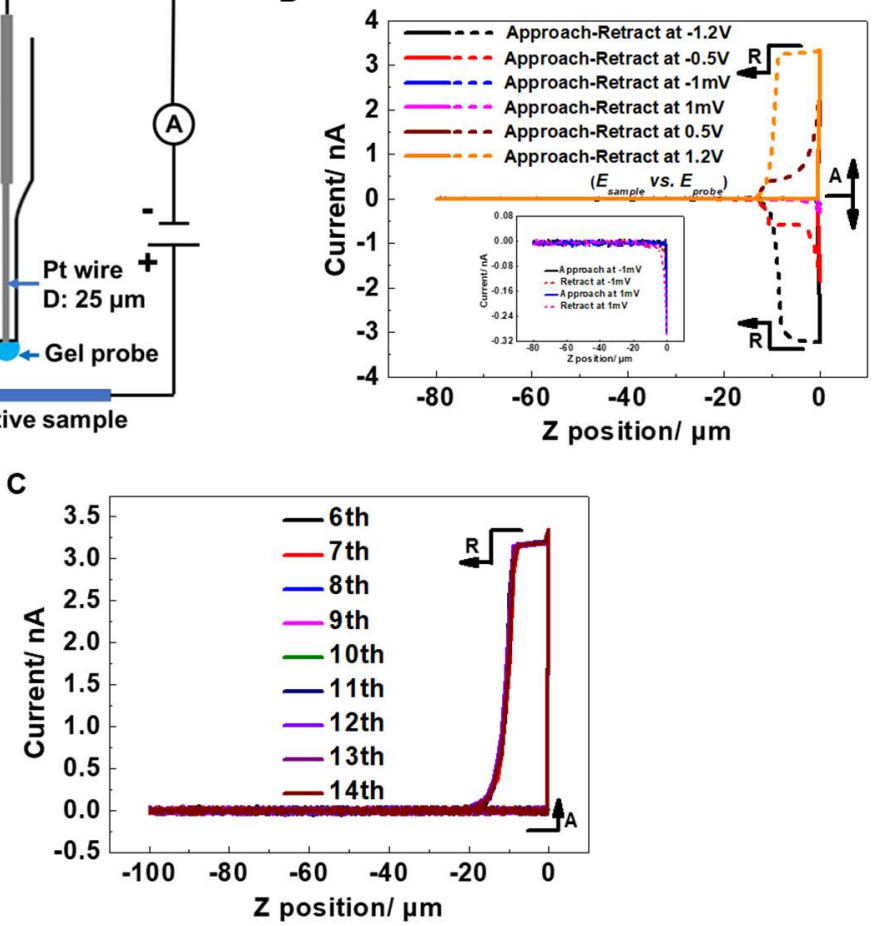

Figure 2 (A) Scheme of SGECM with current feedback; (B) Approach and retract curves of current feedback at different voltage. (C) Multiple approach and retract curves at the voltage of $1.2 \mathrm{~V}$ ( $\left.E_{\text {Sample }} v s . E_{\text {probe }}\right)$ in current feedback.

Approach and retract curves are very informative. First, it helps setting up a feedback loop with appropriate threshold for detecting the contact between the gel probe and the sample. Moreover, it shows that the gel probe can be elastically pressed or stretched in a certain range while keeping contact with the sample. This soft contact allows electrochemical measurements to be carried out at different $z$, which will be explored in the following sections. For conductive samples, current feedback is more sensitive to touching than shear force feedback, thus it is more favourable. It can be technically carried out at almost any voltage, providing high flexibility for choosing the voltage according to the need. Nevertheless, shear force feedback is more versatile, which is still useful for the samples with unknown conductivity, such as partially non-conductive samples.

\section{Determination of lateral physical resolution by $\mathrm{AgCl}$ deposition}

The soft contact as seen from the approach-retract curves may affect the contact area where electrochemical reactions occur, and this may change the lateral physical resolution of SGECM. The most straightforward way to analyse such resolution is permanently marking single sampling points (pixels). This is achieved by locally oxidizing $\mathrm{Ag}$ in the presence of $\mathrm{Cl}^{-}$ forming $\mathrm{AgCl}$, as illustrated in Figure 3A. The gel probe is approached to an $\mathrm{Ag}$ plate with either current or shear force feedback, and then positioned at different $z$. A voltage is then applied to locally oxidize Ag in the contact area. This leaves spots with permanent colour 
change on the sample. Figure 3B shows the SEM image of a typical spot. It is clearly seen that deposition is formed. The deposit is analyzed by EDX and it clearly shows a $\mathrm{Cl}$ peak (Figure 3C). The results confirm the deposition of $\mathrm{AgCl}$ by oxidizing the $\mathrm{Ag}$ plate, thus the physical resolution of SGECM can be derived from the size of the spots.

Figure 4 shows the image of $\mathrm{AgCl}$ spots deposited at $1.2 \mathrm{~V}$ ( $E_{\text {sample }} v s$. $\left.E_{\text {probe }}\right)$ for $300 \mathrm{~s}$. The spot deposited at $z=0$ has diameter of $c a .50 \mu \mathrm{m}$, which is about two times the diameter of the Pt microelectrode and is close to the diameter of the glass shield. At negative $z$ (pulling the gel probe after touching), it is clearly seen that the size of spots decreases (Figure 4A). In contrary, at positive $z$ (pressing the gel probe after touching) the size of spots slightly increases (Figure 4B). The size of spots reflects the area of contact. Considering that the stiffness of the gel is constant for the same probe, the area of contact is proportional to the total force of interaction at the gel/sample interface which is the vector sum of the squeezing/stretching of the gel and the gel/sample adhesion. At negative $z$, the gel is stretched to counterbalance the adhesion, decreasing the total force, while at positive $z$ the gel is squeezed adding the total force. This explains the trend as illustrated in Figure 4C. The results indicate that the lateral physical resolution of SGECM can be tuned in the range of 14 to $78 \mu \mathrm{m}$ by pulling or pressing the same gel probe. This is an important feature of SGECM, which originates from the soft contact between the gel probe and the sample surface. This trend of flexible resolution is reproducible with different gel probes, even though the exact values slightly differ (Figure S4). It should be noted that the spots deposited at intensive pressing of the gel probe $(z>4 \mu \mathrm{m})$ are not homogeneous. Apart from the outer rings that reflect the area of contact, round shape depositions with diameter of around $25 \mu \mathrm{m}$ are seen in the center of the spots. This can be explained by the current distribution, which is more localized when the microelectrode is closer to the sample, similar as direct mode SECM ${ }^{22-24}$. 


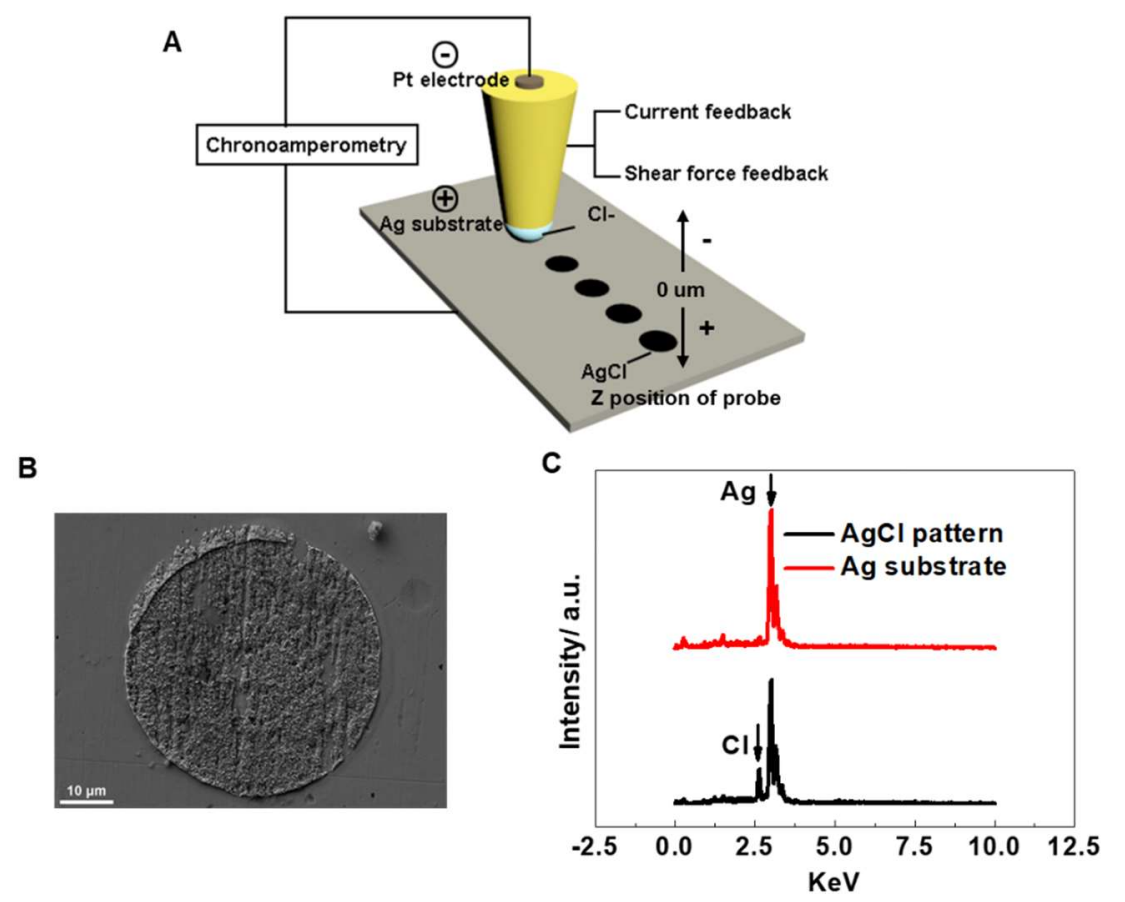

Figure 3 (A) Scheme of $\mathrm{AgCl}$ deposition by current and shear force feedback in SGECM; SEM image (B) and EDX (C) of a typical $\mathrm{AgCl}$ spot deposited at touching position ( $z=0 \mu \mathrm{m})$ of current feedback.

A

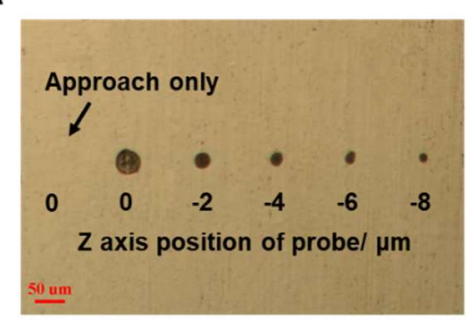

B

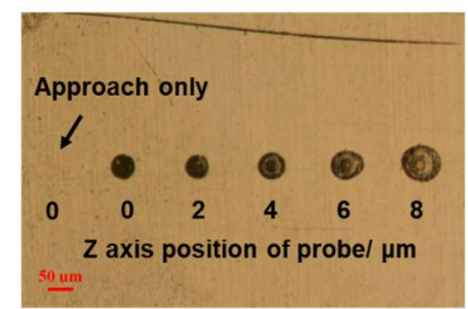

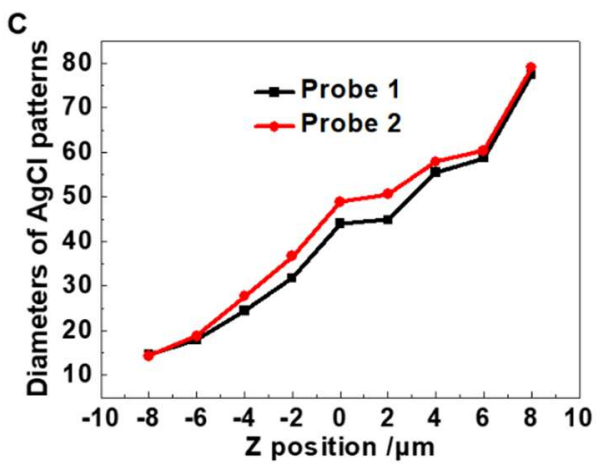

Figure $4 \mathrm{AgCl}$ depositions on $\mathrm{Ag}$ substrate by current feedback $\left(E_{\text {sample }}\left(v s . E_{\text {probe }}\right)=1.2 \mathrm{~V}\right)$ with the gel probe positioned at negative (A), positive (B) $z$ after touching the sample; (C) Size of $\mathrm{AgCl}$ spots as a function of $z$ in current feedback. 
The flexible resolution is also seen when approaching the gel probe by shear force feedback. Considering that the gel is already pressed when the approaching stops (Figure 1C), only pulling (negative $z$ ) is tested. Figure 5 shows that the deposition at $z=0$ has diameter of $c a$. $63 \mu \mathrm{m}$. This is higher than the diameter of that deposited at touching position in current feedback, confirming that the gel probe is indeed pressed. By pulling the gel probe, the size of the spot also decreases, which agrees with the trend observed with current feedback. This is also reproduced with another gel probe (Figure 5B, images shown in Figure S5). The results confirm that the contact area between the gel probe and the sample depends on the pressing/pulling of the gel probe, thus the lateral physical resolution of SGECM is tuneable in the range of tens of microns with the same gel probe. In addition, it further proves that current feedback is more sensitive than shear force feedback for detecting the contact of gel probe on conductive surfaces.

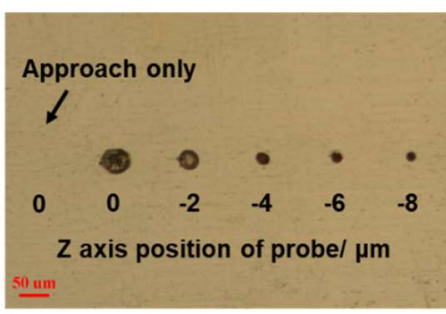

B

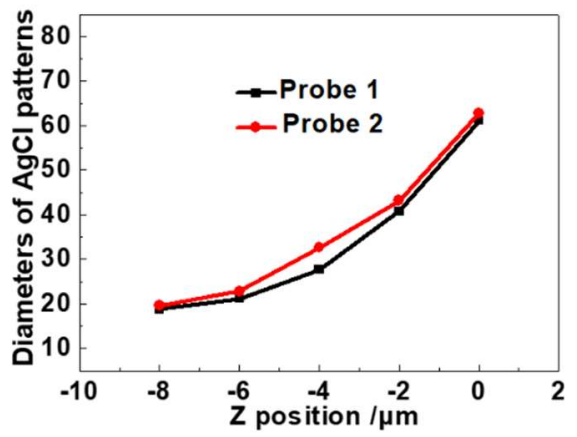

Figure 5 (A) $\mathrm{AgCl}$ depositions on $\mathrm{Ag}$ substrate by shear force feedback, with the gel probe positioned at different negative $z$ after touching the sample; (B) Size of $\mathrm{AgCl}$ spots as a function of $z$ in shear force feedback.

\section{Determination of lateral physical resolution by line scan of periodically shaped samples}

Besides marking single pixels on the Ag plate, another approach for determining lateral physical resolution is to scan periodically shaped samples. Here, a Cu TEM grid stick on a flat steel plate is taken as a reference sample, as demonstrated in Figure6A. The topography of the sample can be retrieved from the touching position coordinates in line scan of SGECM, and the later physical resolution is analyzed by comparing with the results from commercial profilometry measured at the same area.

Figure 6B shows the profile of $\mathrm{Cu}$ grid bars measured from line scan of current feedback SGECM (black curve) and profilometry (red curve). At the edges of the bars, the SGECM profile shows arc and the profilometry shows trapezoid shape. This is because the gel probe has quasi-hemispherical shape, while the profilometry uses cone shape probes. The height of the bars is almost the same for both curves, indicating that SGECM is reliable for topography measurements in $z$ axis. Nevertheless, it should be noted that the profile from SGECM shows "wider" bars than profilometry. This blurring effect is due to the larger diameter of the gel 
probe as compared with the profilometer probe $(d=4 \mu \mathrm{m})$. The difference in the measured width indicates that the lateral physical resolution of SGECM is $c a .46-48 \mu \mathrm{m}$, with the gel probe touching the sample by current feedback (Table S1). The value is similar as that determined from $\mathrm{AgCl}$ deposition at $z=0$. The same probe is also used for line scan SGECM with shear force feedback (Figure 6C). The height of the bars can also be reliably measured, but the width appears even larger than in current feedback SGECM, suggesting that the lateral physical resolution is poorer. The calculated lateral physical resolution is $c a$. $62.5 \mu \mathrm{m}$ (Table S1). This confirms that the gel probe is already pressed at touching position of shear force feedback, which agrees with the trend observed in approach curves (Figure 1C) and $\mathrm{AgCl}$ depositions (Figures 4 and 5).

Apart from the touching coordinates that reflect the topography, the steady state current under a constant voltage is also recorded when the gel probe touches the sample, which is an indication of reactivity of the sample. From Figure 6, it is clearly seen that the current measured on $\mathrm{Cu}$ grid is much higher than that on the Fe substrate in both current and shear force feedback. The redox behaviour of $\mathrm{Cu} / \mathrm{Cu}^{2+}$ can be seen from the peaks in cyclic voltammetry, while Fe is more inert perhaps due to the passivation (Figure S6). As a result, the current in SGECM may differentiate the two metals based on their different reactivity. It is interesting to see that the current measured with shear force feedback is almost 10 times higher than that measured with current feedback. Considering that the contact area in shear force feedback is only less than 2 times higher than that in current feedback, there should be other factors causing this significant difference. One possibility is that the distance between the Pt microelectrode and the sample is reduced in shear force feedback due to the pressing of the gel. This may facilitate the redox of $\mathrm{Cu}$, like in the sample generation-tip collection mode SECM $^{25,26}$. 
A

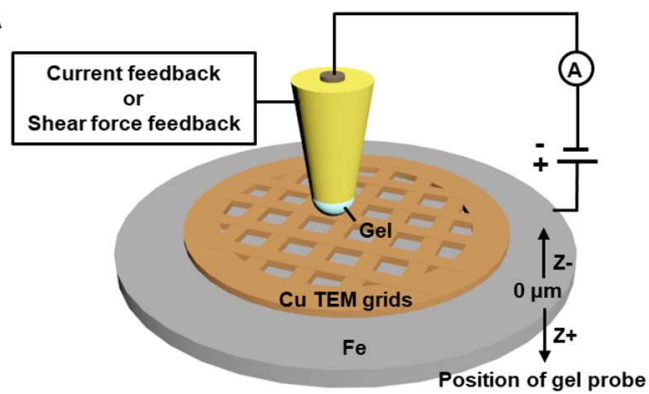

B

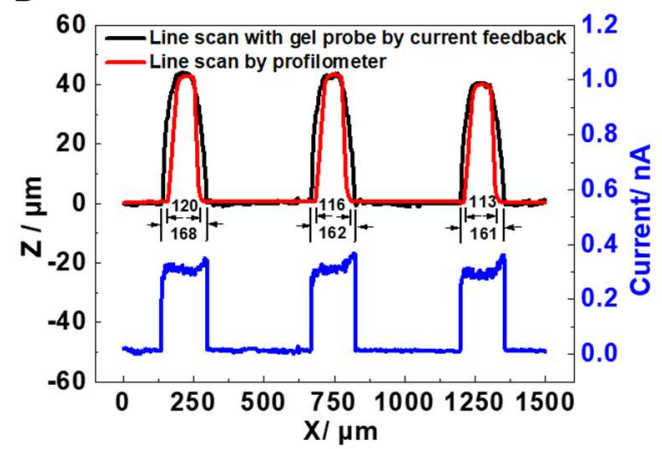

C

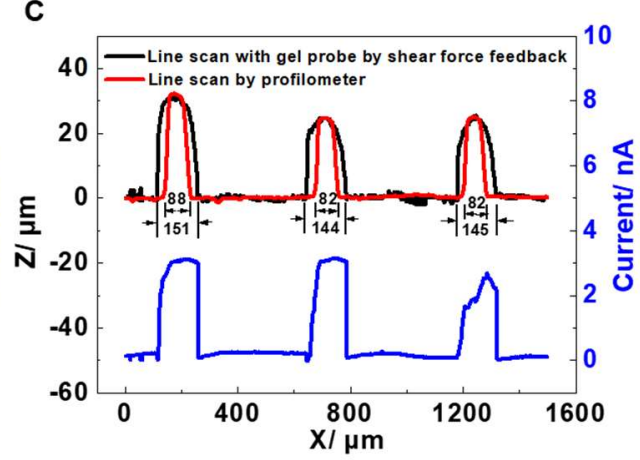

Figure 6 (A) Scheme of line scan on Cu TEM grid/Fe plate by current and shear force feedback SGECM; Line scan of profile and steady state current by current (B) and shear force

(C) feedback. The data were recorded at touch position with $E_{\text {sample }} v s$. $E_{\text {probe }}=0.5 \mathrm{~V}$ after waiting for $3 \mathrm{~s}$.

Since the topography is measured based on the touching position in SGECM, it is obviously only possible to determine the physical resolution at $z=0$ from the profile. Nevertheless, the current response in line scan allows examining the flexible resolution feature of SGECM by pulling or pressing the gel probe. Figure 7 compares the topography with steady state current at different $z$ measured at the edge of a $\mathrm{Cu}$ grid bar using current feedback. At $z=0$ (Figure 7A), the current step fits well with the profile step in lateral position, indicating that the two measurements have the same physical resolution which is trivial to understand. By pulling the probe to $z=-2 \mu \mathrm{m}$ (Figure 7B), the current decreases before the touching position when scanning from the $\mathrm{Cu}$ bar to the Fe substrate. This suggests that pulling the gel probe would reduce the contact area thus increase the physical resolution of the measurement. In contrary, when pressing the probe to $z=4 \mu \mathrm{m}$ (Figure 7C), high current is still measured even when the probe laterally leaves the $\mathrm{Cu}$ bar at touching position. This is likely due to the pressing of the probe that increases the contact area and re-includes the $\mathrm{Cu}$ part. The pressing of the probe also yields an increase in the absolute value of current on $\mathrm{Cu}$, which has been discussed in the previous paragraph. Similar trends are also observed with shear force feedback (Figure S7). The results provide another proof for the flexible resolution feature of SGECM. 
A

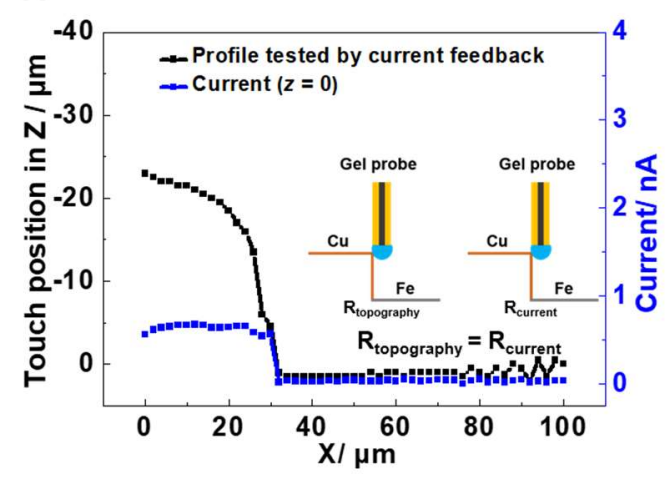

B

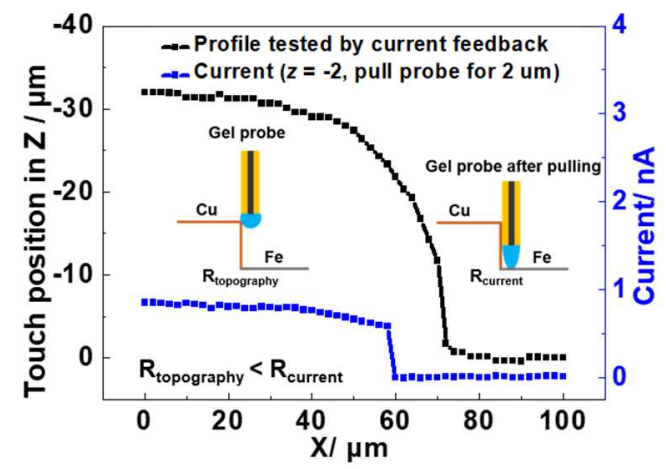

C

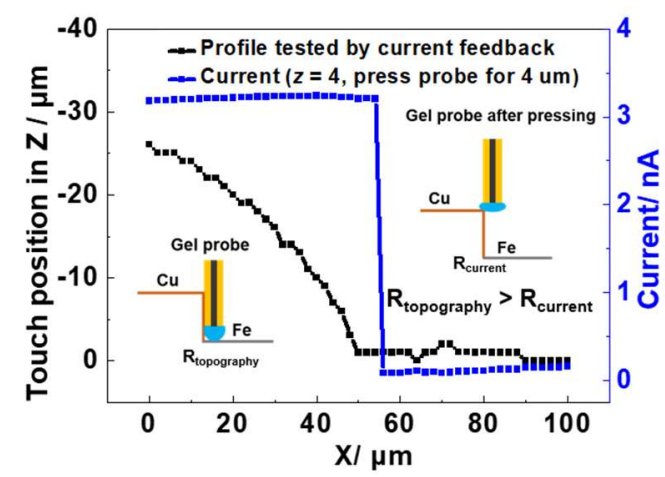

Figure 7 Line scan of profile and steady state current on $\mathrm{Cu}$ TEM grid/Fe plate by current feedback SGECM. The current is recorded at $z=0$ (A), -2 (B) and 4 (C) $\mu \mathrm{m}$ at $E_{\text {sample }} v$ s. $E_{\text {probe }}=0.5 \mathrm{~V}$ after waiting for $3 \mathrm{~s}$.

From the results above, it is clear that the current feedback is more sensitive to touching than shear force feedback, because the gel is already pressed at the touching position in the latter. Therefore, it is more favourable to use current feedback for approaching the gel probe to the sample. Nevertheless, current feedback requires the whole sample to be conductive, while shear force feedback does not have this restriction. For example, Figure 8A illustrates $\mathrm{Au}$ interdigitated electrodes with one electrode plated with $\mathrm{Cu}$. In this case, the sample consists of $\mathrm{Au}$ and $\mathrm{Cu}$ that are conductive, as well as insulating ceramic between them. Thus it can only be measured with shear force feedback SGECM. Figure $\mathbf{8 B}$ shows the profile and steady state current of a line scan. Since the thickness of Au interdigitated electrodes is negligible, only $\mathrm{Cu}$ plating bars of $c a .10 \mu \mathrm{m}$ height are visible in the profile. This is in agreement with the profilometry (Figure S8). Meanwhile, current signals could reveal both $\mathrm{Cu}$ and $\mathrm{Au}$, as well as the insulating ceramic. At $E_{\text {sample }} v s$. $E_{\text {probe }}=1.0 \mathrm{~V}, \mathrm{Cu}$ shows higher current response than $\mathrm{Au}$, which can be explained by its higher electrochemical activity towards oxidation (Figure S9). As expected, current is almost 0 (with noise) when the gel probe touches the ceramic part of the sample. This example demonstrates the versatility of shear force feedback SGECM, which can be used in measuring any sample with unknown conductivity. 

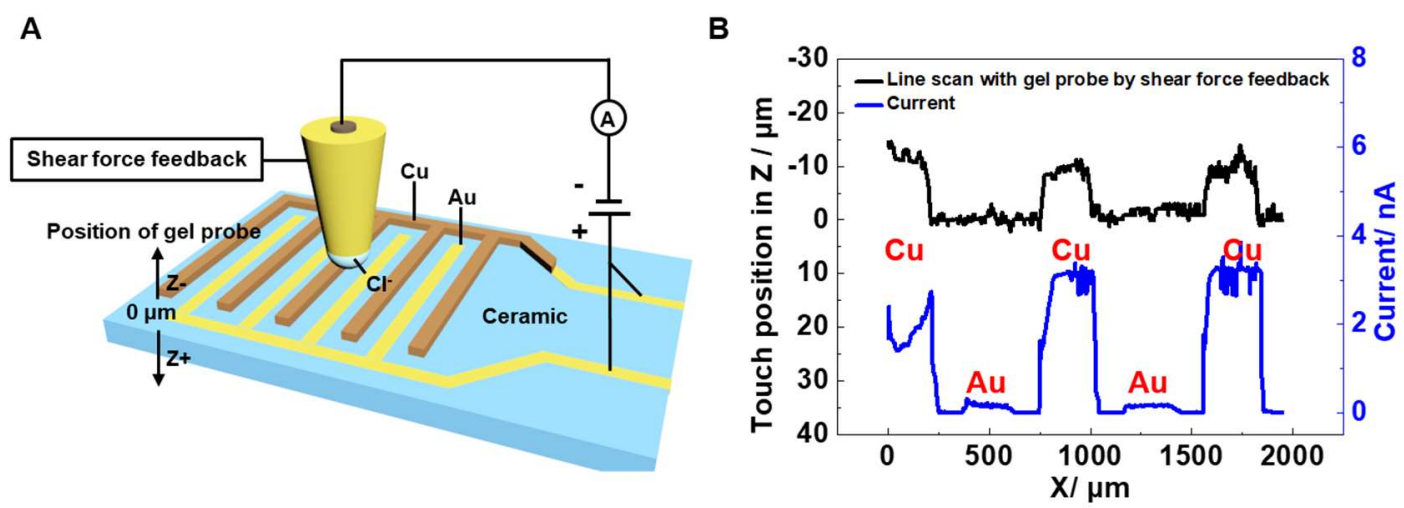

Figure 8 (A) Scheme of line scan on Cu-plated Au interdigitated electrodes by shear force feedback SGECM; (B) Line scan of profile and steady state current $\left(E_{\text {sample }} v s . E_{\text {probe }}=1.0 \mathrm{~V}\right)$ measured at touching position by shear force feedback.

\section{Conclusion}

In conclusion, lateral physical resolution of SGECM is quantitatively analyzed from two aspects: the size of single sampling spots, and the line scan of periodically shaped reference samples. The former is achieved by locally oxidizing $\mathrm{Ag}$ to $\mathrm{AgCl}$ by the gel probe, leaving permanent colour change on the sample surface. The latter is carried out on a $\mathrm{Cu}$ TEM grid stick on a Fe plate. From both experiments, it is seen that current feedback has higher lateral physical resolution than shear force feedback with the same gel probe. This is because current feedback is more sensitive to the contact between the gel probe and the sample, and the gel is already pressed when shear force feedback indicates the touching. Therefore, current feedback is more favourable for conductive samples, but shear force feedback is more versatile especially to the samples with unknown conductivity. For the probe fabricated by electrodepositing chitosan on a $25 \mu \mathrm{m}$ Pt disk electrode with $R_{g} \approx 2$, the lateral physical resolution at touching position $(z=0)$ is $c a .50 \mu \mathrm{m}$ for current feedback and $c a .67 \mu \mathrm{m}$ for shear force feedback. More importantly, due to the soft nature of the gel, the physical resolution can be flexibly tuned from 14 to $78 \mu \mathrm{m}$ by pulling or pressing the gel probe for a certain distance after touching the sample. This is a useful feature that opens the possibility for multi-resolution imaging or patterning by SGECM using the same gel probe.

\section{Acknowledgement}

The authors gratefully acknowledge financial support from CNRS (MOMENTUM project 2018-2010) and Université de Lorraine (Projet Structurant 2017-2018). The authors also acknowledge Andreï Lecomte for the SEM and EDX measurements.

\section{References}

(1) Filotás, D.; B. M. Fernández-Pérez; Izquierdo, J.; Kissa, A.; Nagy, L.; Nagy, G.; Soutoc, R. M. Corros. Sci. 2017, 129, 136. 
(2) Zhang, Q. H.; Ye, Z. N.; Zhu, Z. J.; Liu, X. Y.; Zhang, J. Q.; Cao, F. H. Corros. Sci. 2018, 139, 403.

(3) Ventosa, E.; Wilde, P.; Zinn, A. H.; Trautmann, M.; Ludwig, A.; Schuhmann, W. Chem. Commun. 2016, 52, 6825.

(4) Ventosa, E.; Schuhmann, W. Phys. Chem. Chem. Phys. 2015, 17, 28441.

(5) Bulter, H.; Peters, F.; Schwenzel, J.; Wittstock, G. Angew. Chem. Int. Ed. Engl. 2014, 53, 10531 .

(6) Ventosa, E.; Madej, E.; Zampardi, G.; Mei, B.; Weide, P.; Antoni, H.; Mantia, F. L.; Muhler, M.; Schuhmann, W. ACS Appl. Mater. Interfaces 2017, 9, 3123.

(7) Yan, R.; Ghilane, J.; Phuah, K. C.; Truong, T. N. P.; Adams, S.; Randriamahazaka, H.; Wang, Q. J Phys. Chem. Lett. 2018, 9, 491.

(8) Fernández, J. L.; Bard, A. J. Anal. Chem. 2003, 75, 2967.

(9) Botza, A.; Clausmeyera, J.; Öhla, D.; Tarneva, T.; Franzenb, D.; Turekb, T.; Schuhmann, W. Angew. Chem. Int. Ed. 2018, 57, 12285.

(10) Bentley, C. L.; Kang, M.; Unwin, P. R. J. Am. Chem. Soc. 2017, 139, 16813.

(11) Kuss, S.; Trinh, D.; Mauzeroll, J. Anal. Chem. 2015, 87, 8102.

(12) Solda, A.; Valenti, G.; Marcaccio, M.; Giorgio, M.; Pelicci, P. G.; Paolucci, F.; Rapino, S. ACS Sens. 2017, 2, 1310.

(13) Welle, T. M.; Alanis, K.; Colombo, M. L.; Sweedler, J. V.; Shen, M. Chem. Sci. 2018, 9 , 4937.

(14) Shevchuk, A. I.; Frolenkov, G. I.; Sánchez, D.; James, P. S.; Freedman, N.; Lab, M. J.; Jones, R.; Klenerman, D.; Korchev, Y. E. Angew. Chem. 2006, 118, 2270.

(15) Müller, M.; Mills, R. A.; Pearce, R. B.; Milton, J. A.; Statham, P. J.; Lloyd, J. R.; Mujahid, A.; Denuault, G. Geomicrobiol. J. 2014, 31, 373.

(16) Atesyan, A.; Belhadj, O.; Combellas, C.; Kanoufi, F.; Rouchon, V.; Noël, J. M. ChemElectroChem 2019, 6, 779.

(17) Thatenhorst, D.; Rheinlaender, J.; Schaffer, T. E.; Dietzel, I. D.; Happel, P. Anal. Chem. 2014, $86,9838$.

(18) Dang, N.; Etienne, M.; Walcarius, A.; Liu, L. Electrochem. Commun. 2018, 97, 64.

(19) Liu, L.; Etienne, M.; Walcarius, A. Anal. Chem. 2018, 8889.

(20)Etienne, M.; Anderson, E. C.; Evans, S. R.; Schuhmann, W.; Fritsch, I. Anal. Chem. 2006, $78,7317$.

(21) Etienne, M.; Lhenry, S.; Cornut, R.; Lefrou, C. Electrochim. Acta 2013, 88, 877.

(22) Liu, L.; Toledano, R.; Danieli, T.; Zhang, J. Q.; Hu, J. M.; Mandler, D. Chem. Commun. 2011, 47, 6909 .

(23) Liu, L.; Mandler, D. Electrochem. Commun. 2014, 48, 56.

(24) Sarkar, S.; Mandler, D. ChemElectroChem 2017, 4, 2981.

(25) Martin, R. D.; Unwin, P. R. Anal. Chem. 1998, 70, 276.

(26)Minguzzi, A.; Alpuche-Aviles, M. A.; López, J. R.; Rondinini, S.; Bard, A. J. Anal. Chem. 2008, $80,4055$. 